all the other sequences of the formation of life still need much investigation in order to provide us with a comprehensive picture. However, as more data become available from year to year we may be justified in hoping that in the not too distant future at least some of the problems will be resolved.

What we can safely say now is that nature is controlled by the entirety of its laws, that these laws interact with each other, and that the scientific world emerges as a unity in which each particle has its own well defined place, time, and function.

Received: December 29, 1972 [A 944 IE] German version: Angew. Chem. 85,422 (1973)

[1] The Works of Francis Bacon, V. 1. Reever, London 1879.

[2] R. Descartes: Oeuvres philosophiques. Aime et Martin, Paris 1838.

[3] F. Redi: Esperienze intorno alla generazione degl'insetti. 1668.

[4] L. Pasteur, C. R. Acad. Sci. Paris 50, 303 (1860)

[5] L. Pasteur, C. R. Acad. Sci. Paris 50, 675 (1860)

[6] L. Pasteur, C. R. Acad. Sci. Paris 50, 849 (1860)

[7] L. Pasteur, C. R. Acad. Sci. Paris 51, 348 (1860).

[8] L. Pasteur, Ann. Sci. Nat. 5, 16 (1861).

[9] L. Pasteur, Ann. Chim. Phys. (3), 5, 64 (1862).

[10] L. Pasteur, C. R. Acad. Sci. Paris 56, 734 (1863).

[11] Francis Darwin: The Life and Letters of Charles Darwin. Murray, London 1887, Vol. 3, Vol. 18

[12] S. L. Miller, J. Amer. Chem. Soc. 77, 2351 (1955).

[13] A. Gulick, Amer. Sci. 43, 749 (1955).

[14] J. J. Scott, Biochem. J. 62, 6 P (1956).

[15] J. Oró and C. L. Guidry, Nature 186, 156 (1960).

[16] J. Oró and A. Kimball, Arch. Biochem. Biophys. 85, 115 (1959).

[17] J. Oró and A. Kimball, Arch. Biochem. Biophys. 94, 217 (1961).

[18] J. Oró and S. Kamat, Nature 190, 442 (1961).

[19] J. Oró, Biochem. Biophys. Res. Commun. 2, 407 (1960).

[20] C. Palm and M. Calvin, J. Amer. Chem. Soc. 89, 2115 (1962).

[21] C. Ponnamperuma, C. Sagan, and R. Mariner, Nature 199, 222

[22] A. Schwartz and C. Ponnamperuma, Nature 218, 443 (1968).

[23] A. Bar-Nun, N. Bar-Nun, S. H. Bauer, and C. Sagan in R. Buvet and C. Ponnamperuma: Chemical Evolution and the Origin of Life. NorthHolland Publ. Co., Amsterdam 1971, Vol. 1, p. 114.
[24] C. N. Mathews and R. E. Moser, Proc. Nat. Acad. Sci. USA 56 1087 (1966).

[25] G. Schramm in S. W. Fox: The Origins of Prebiological Systems Academic Press, New York 1965, p. 299

[26] A. 1. Oparin: Life, Its Nature, Origin, and Development. Oliver and Boyd, Edinburgh 1961, p. 47

[27] A. I. Oparin and K. B. Serebrovskaya, Dokl. Akad. Nauk SSSR $148,943(1963)$.

[28] T. N. Evreinova, P. Pogosova, T. Tsukawara, and T. Lapinova, Nauch. Dokl. Vyssh. Shk. 1,159 (1962).

[29] M. Calvin, Science 130, 1170 (1959).

[30] J. D. Bernal: The Physical Basis of Life. Routledge and Kegan Paul, London 1951.

[31] M. Paecht-Horowitz, J. Berger, and A. Katchalsky, Nature 228, $636(1970)$.

[32] R. Lewinsohn, M. Paecht-Horowitz, and A. Katchalsky, Biochim. Biophys. Acta 140, 24 (1967).

[33] M. Paecht-Horowitz and A. Katchalsky, Biochim. Biophys. Acta 140,14 (1967).

[34] M. Paecht-Horowitz in $R$. Buvet and C. Ponnamperuma: Chemical Evolution and the Origin of Life. North-Holland Publ. Co., Amsterdam 1971, Vol. 1, p. 245.

[35] I. Prigogine in R. J. Donnelly, R. Herman, and I. Prigogine: NonEquilibrium Thermodynamics, Variational Techniques and Stability. University of Chicago Press, Chicago 1966, p. 3.

[36] A. G. Cairns-Smith, J. Theor. Biol. 10, 53 (1966)

[37] $M$. Eigen, Naturwissenschaften 58, 465 (1971).

[38] F. Lipman, Advan. Enzymol. I, 154 (1941).

[39] M. Paecht-Horowitz and A. Katchalsky. J. Mol. Evol. in press.

[40] S. W. Fox, K. Harada, and J. Kendrick, Science 129, 1221 (1959).

[41] S.W. Fox, Science 132, 200 (1960).

[42] R. S. Young in S.W. Fox: The Origins of Prebiological Systems. Academic Press, New York 1965, p. 350.

[43] H. Bénard, Rev. Gen. Sci. Pures Appl. 11, 1261 (1900).

[44] H. Bénard, Rev. Gen. Sci. Pures Appl. 11, 1309 (1900).

[45] H. Bénard, Ann. Chim. Phys. 23, 62 (1901).

[46] H. L. Koschmieder in R.J. Donnelly, R. Herman, and I. Prigogine: Non-Equilibrium Thermodynamics, Variational Techniques and Stability. University of Chicago Press, Chicago 1966, p. 172.

[47] A. M. Turing, Trans. Roy. Soc. (London) B 237, 37 (1952).

[48] A. M. Zhabot inskii, Biofizika 9, 306 (1964).

[49] A. M. Zhabotinskii, Dokl. Akad. Nauk SSSR 157, 392 (1964)

\title{
Molecular Hysteresis and Its Cybernetic Significance
}

\author{
By Eberhard Neumann ${ }^{[*]}$
}

The general foundations for a thermodynamic analysis of hysteresis phenomena in solutions and suspensions of polyelectrolyte systems are presented using examples of molecular hysteresis in biopolymers and membranes. The fundamental cybernetic significance of metastable states and molecular hysteresis for a physical interpretation of phenomena of life such as memory recording and biological rhythms is discussed.

\section{Introduction}

"I believe that the ultimate goal of biological study is to 'translate' the phenomena of life into meaningful physical concepts".- Aharon Katchalsky $\left.{ }^{* *}\right]$

[*] Priv.-Doz. Dr. E. Neumann

Max-Planck-Institut für Biophysikalische Chemie

34 Göttingen-Nikolausberg, Postfach 968 (Germany)

[**] "Wer ist's", Nachr. Chem. Techn. 20 (13), 247 (1972)
Modern physical chemistry of biological macromolecules has its roots in classical colloid chemistry. Because of their colloid properties high molecular weight constituents of biological cells and tissues have been called biocolloids. However, it was soon recognized that many cellular components thus classified were not colloidal aggregates of small molecules but large polymers of colloidal dimensions $^{[1]}$. 
The majority of biological macromolecules are linear polymer chains whose constituent monomer residues are electrically charged under physiological conditions of $\mathrm{pH}$ (about $\mathrm{pH} 7$ to $\mathrm{pH} 8$ ) and ionic strength (about 0.15 mol/l). Thus, for instance, all nucleic acids and acidic mucopolysaccharides are strong polyelectrolytes. The "surfaces" of many structural proteins, enzymes and biomembranes are densely covered with electrically charged groups and hence may be considered as polyelectrolyte systems.

In recent decades increased attention has been devoted to the electrochemical properties of biopolymers and biopolyelectrolyte organizations such as membranes. It has been recognized that various aspects of the specific organization and function of these cellular components are determined by the electrical interactions between them and their environment. Thus changes in structure and function of such systems may be regulated and controlled by electrical influences of the environment ${ }^{[2]}$. This cybernetic aspect gains particular interest in connection with another remarkable property of many "biocolloids": the capability of developing long-lived metastable states.

It is known from colloid chemistry that colloids of high electric charge may frequently exist in states that are not thermodynamically stable; such systems may undergo irreversible phase changes ${ }^{[3]}$. Thus it is not surprising that polyelectrolyte organizations of biopolymers and biomembranes are also capable of developing long-lived metastable states. In a variety of cases such metastabilities are reflected in pronounced hysteresis phenomena.

Perhaps the most familiar examples of long-lived metastabilities are the hysteresis loops displayed by ferromagnetic materials. But hysteresis phenomena are also seen in other fields of physics and chemistry; for instance, in meltingcrystallization cycles of ammonium salts or in adsorptiondesorption processes on porous materials. In these cases hysteretic behavior has been macroscopically interpreted in terms of domain and pore structures ${ }^{[4]}$.

The term hysteresis was coined by Ewing in order to describe the (history-dependent) reaction of magnets to changes of the external electric field ${ }^{[5]}$. The word is derived from the Greek $\dot{\sigma} \sigma \tau \rho \varepsilon \dot{\omega}=$ to lag behind; for instance, in a magnetization-demagnetization cycle the magnetic polarization lags behind the applied magnetic field intensity. In contrast to the classical macroscopic hysteresis phenomena in and on condensed phases, microscopic hysteresis of individual molecules in homogeneous solution has been discovered only recently. In 1956, Cox, Jones, Marsh, and Peacocke found that the potentiometric acid-base titration of ribosomal ribonucleic acid (rRNA) does not yield an equilibrium curve, but exhibits hysteresis in the degree of proton binding as a function of the $\mathrm{pH}$ value ${ }^{[6]}$. It was later shown that the rRNA hysteresis is an intramolecular phenomenon ${ }^{[7]}$.

The result of a spectrophotometric acid-base titration of rRNA is shown in Figure 1. The hysteresis loop is practically time-independent (see Section 2) and can be traced several times. The direction of cycling, however, is fixed: titration of a neutral solution with acid always yields the lower branch, subsequent titration of the acidic solution with base always follows the upper branch of the limiting loop. Within the boundary loop we may obtain scanning

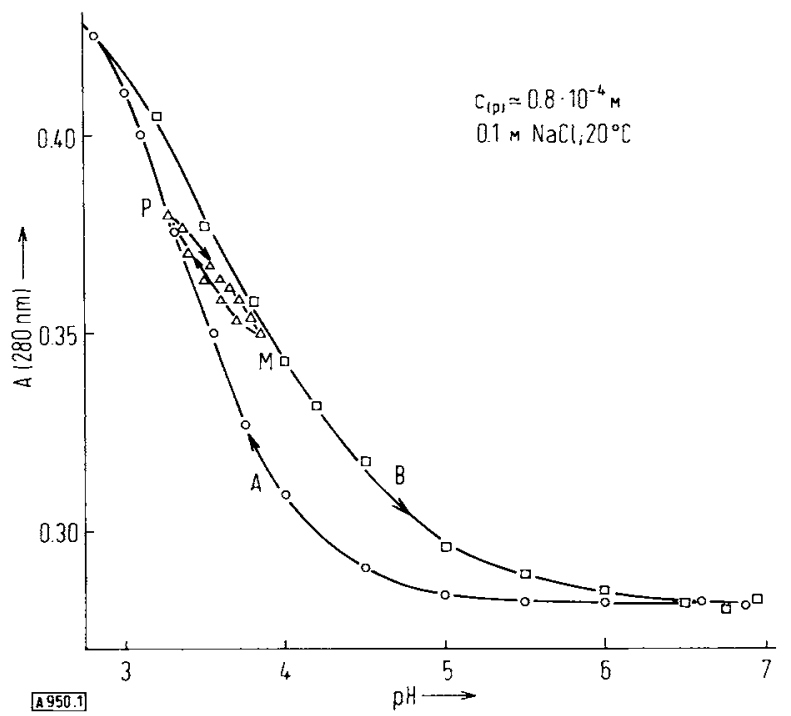

Fig. 1. Spectrophotometric titration of ribosomal RNA. Absorbance (A) at $280 \mathrm{~nm}$ as a function of $\mathrm{pH} ; O$, acid titration (curve $\mathrm{A}$ ); $\square$, subsequent base titration (curve B); $\Delta$, scanning loop. After [7].

curves similar to those observed in magnetic hysteresis. Because these inner curves reflect intermediary states of the system, scanning can in principle be used to scrutinize these states. The analysis of scanning curves is therefore a (novel) method of studying structural subunits in biopolymers such as rRNA in solution ${ }^{[8]}$.

Numerous examples of hysteresis in solution and suspension are now known. Less numerous, however, are attempts to analyze these nonequilibrium phenomena and to elucidate the molecular origin of the underlying metastabilities. Indeed, for some time, hysteresis seems to have been considered by physicists and chemists as a curiosity that had to be avoided. That cycling of the loop is directional appears inconsistent with the time-independence of stable hysteresis loops. Directionality and changes with time are properties of irreversible (natural) processes whereas timeindependence is the criterion for the limiting case of equilibrium (or of stationary states). Time-independent irreversibility, as reflected in long-lived hysteresis, apparently could not be treated either by the methods of classical thermostatics or by those of nonequilibrium thermodynamics.

It is the merit of Aharon Katchalsky to have contributed to a conceptual clarification of this apparent contradiction and, furthermore, to have recognized the important role which metastabilities and hysteresis may play in central phenomena of life, such as memory recording or biological rhythms ${ }^{[9,10]}$. Hysteresis is a well known expression of physical memory; thus modern computer technology utilizes hysteresis cycles of ferromagnetic and ferritic materials as memory devices for information storage.

In biology the mechanism of memory recording is still an unsolved problem. We may, however, conclude from biochemical and pharmacological studies that in the multistage process of biological memory formation there could plausibly be a physical step which is based on the directionality of hysteresis in macromolecules or membranes ${ }^{[11]}$. As a further dynamic aspect of hysteresis, circulation around a hysteresis loop presents in principle a possibility of producing periodicity on a molecular level by chemicodiffusional coupling ${ }^{\{0,12\}}$. This recognition is of great interest not only for the dynamics of enzyme catalysis 
but also for the attempts to interpret biological rhythms ${ }^{[13]}$ in terms of oscillatory chemical reactions.

Particularly challenging is the observation of hysteresis in nerve membranes ${ }^{[14.15]}$. There is no doubt as to the fundamental role of electrochemical excitability of membranes for communication and control in all living organisms. However, the elementary processes of generation and conduction of bioelectric impulses are not well understood. It is remarkable that, according to Adam's molecular theory of nerve excitability, the release of action potentials proceeds via short-lived metastable states of the nerve membrane ${ }^{[16]}$.

These short remarks may indicate that the study of metastability and molecular hysteresis, as observed in macromolecules and membranes in solution and suspension, may be classed as an effort to "translate" some "biological phenomena to physical-mechanistic concepts" ${ }^{\text {"I9] }}$.

\section{Thermodynamics of Hysteresis}

\subsection{Hysteresis and Metastability}

In order to understand the special dynamic properties of hysteresis, we must delve into the nature of metastable states. Wherever long-lived hysteresis is observed, it indicates thermodynamically metastable states and cooperative nonequilibrium transitions. As is the case for macroscopic hysteresis phenomena in and on condensed phases, molecular hysteresis in solution and suspension also seems to be linked with domain structures. A domain may be defined as a structural unit or a structural region whose elementary units are able to change their state cooperatively, i.e. as a whole. Cooperative structural transitions in biopolymers and membranes may be thermodynamically classified as "diffuse" phase transitions and may be treated to a good approximation as first-order phase transitions ${ }^{[17-20]}$.

It is frequently observed that phase changes encounter energy barriers which may hinder nucleation or/and propagation processes. These barriers must be reduced (e.g. by establishing nucleation centers) if the system is to pass through equilibrium states during the transition. If this is not possible under given experimental conditions, the system will pass instead into metastable states. Superheating and supercooling phenomena in thermal phase changes are manifestations of such transition barriers.

Thermodynamically the transition behavior of cooperative systems with metastabilities may be described by a state function $y(x)$ of the van der Waals type ${ }^{[21]}$. Figure $2 \mathrm{a}$ schematically shows such a function for the transformation of a domain between two states I and II. Between the two stable branches of this state function there is a (dotted) non-realizable range of instability. The equilibrium path, between $\mathrm{B}$ and $\mathrm{D}$ for $\mathrm{I} \rightleftharpoons \mathrm{II}$, may be traced at $x=x(\mathrm{G})$. If sufficiently high energy barriers prevent an equilibrium transition, changes in state can only occur via metastable regions. State $I$ is metastable between $B$ and 2 (region III), state II is metastable between $\mathrm{D}$ and 4 (region IV). The phase changes are unidirectional and irreversible, and take place at different $x$-values. At $x(\mathrm{I})$ the transition from I to II (between 2 and 3) occurs; the transition from II to I (between 4 and 1 ), however, occurs at $x$ (II). Thus, the irreversible phase changes fix a direction of cycling the hysteresis loop: $1 \rightarrow 2 \rightarrow 3 \rightarrow 4 \rightarrow 1$.

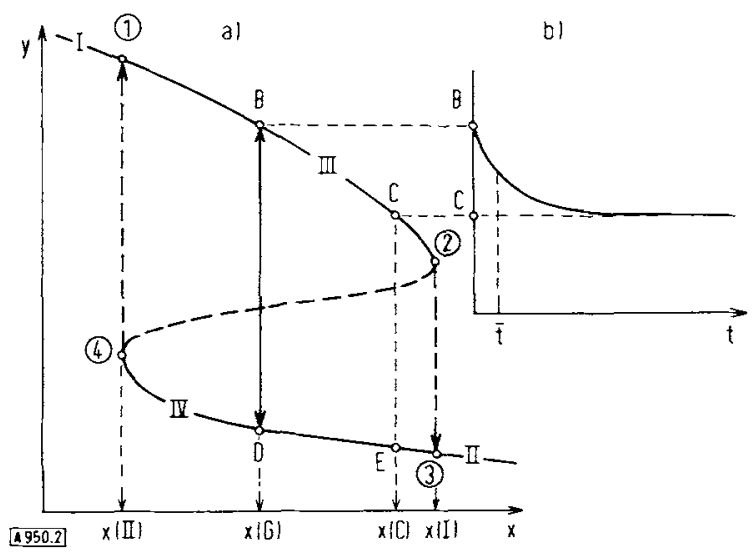

Fig. 2. a) Schematic representation of a phase transition: I, II stable states; III, IV, metastable states. $y$, internal parameter, e.g. volume or extent of reaction; $x$, external parameter, e.g. temperature or $\mathrm{pH}$ value. Cf. text. b) Time course $y(t)$ after a change from $x(G)$ to $x(C)$; see text

In order to obtain hysteresis it is not necessary that both states I and II be metastable. It is sufficient that only one state develops metastability, e.g. state II in the region IV. In this case the transition IV to I would be irreversible whereas the change from I to II would occur along the equilibrium path at $x(\mathrm{G})$.

It may be seen from Figure 2 that in the hysteresis region between the transition points the internal parameter $y$ is not a single-valued function of the external variable $x$. Thus, in contrast to equilibrium curves, hysteresis implies two values for $y$ at one given value of $x$. Depending on the initial state, I or II, and depending on the direction of the change in $x$, the system may be either on the upper or on the lower branch of the $y(x)$ cycle. In the region of hysteresis the system "remembers" the history of tracing, the path by which the actual state change has been reached. It is this simple type of memory of path that distinguishes hysteresis from thermodynamic equilibrium. A state of equilibrium is "memoryless". Equilibrium may be reached by various routes; but once equilibrium is established, the system "forgets" preceding events. The memoryless state of equilibrium is the dead end of all directional, irreversible processes ${ }^{[9]}$.

Abrupt, irreversible transitions characterize the dynamic behavior of a single domain. In an assembly of domains which have different stabilities, the distribution of transition points (along the $x$-axis) leads to an apparently smooth hysteresis curve, in which the consecutive irreversible transitions of individual domains cannot be distinguished (within experimental accuracy). Scanning curves may arise in systems having domains of different size and stability. Whereas hysteresis is a general indicator of cooperativity and metastability, scanning curves imply the existence of "polycrystallinity" or domain structures. Thus, the very observation of scanning curves classifies the rRNA molecule as such a "polycrystalline" multidomain system. 


\section{2. "Time-Independence" of Stable Hysteresis}

As mentioned above, hysteresis is ascribed to thermodynamically metastable states ${ }^{[22]}$, the lifetime of which may differ from one system to another.

Figures $2 \mathrm{a}$ and $2 \mathrm{~b}$ illustrate how a hysteresis domain may react to a change of the environment. Suppose the system is in state $I$ at point $B$ and $x$ is changed from $x(G)$ to $x(C)$. In typical examples one observes that the internal parameter, after an initial fast change (halftime $\bar{t}$ ), levels off to an apparently constant value C. If, within the limits of experimental accuracy, this value remains constant for long observation times $t \gg \bar{t}$, we attribute longevity to the underlying metastability. When the apparent temporal invariance holds only for short observation times, one speaks of short-lived metastable states. In the limit of an extremely small transition probability for the conversion from $C$ to equilibrium at $E$, the extent of transition $\xi$ remains apparently constant (i.e. $\mathrm{d} \xi / \mathrm{d} t=0$ ) in spite of a non-zero affinity $A$. Hence the domain state $\mathrm{C}$ is comparable with a state of hindered equilibrium $(A>0 ; \mathrm{d} \xi / \mathrm{d} t=0)$ such as the (metastable) mixture of $\mathrm{H}_{2}$ and $\mathrm{O}_{2}$ at room temperature, which reacts immeasurably slowly to give the thermodynamically stable $\mathrm{H}_{2} \mathrm{O}$ vapor. It is a consequence of the apparent time-independence, that long-lived metastable states can be described by thermodynamic state functions, in the same way as equilibrium states.

The behavior of a domain is apparently reversible as long as external perturbations are restricted to the regions of metastability. If in our example the system at $B$ is subjected to a displacement of $x$ from $x(G)$ to $x(C)$ and then back to $x(G)$, the domain will first change to point $C$ and then will return from $\mathrm{C}$ to $\mathrm{B}$. If, however, the external change proceeds beyond $x(\mathrm{I})$, so that the irreversible transition to state II can occur, then-due to the directionality of the transition $2 \rightarrow 3$ - the system remains in state II, even when the original condition of $x(\mathrm{G})$ is reestablished. Only a change of $x$ in the opposite direction, going beyond $x(\mathrm{II})$, can bring the system back to its original condition in state $I$ at point $B$. This nonequilibrium behavior means that such a domain may serve as a matrix for a permanent record of directed chemical or physical changes of the environment.

A cyclic variation of $x$, extending beyond both transition points $x(\mathrm{I})$ and $x(\mathrm{II})$, generally leads to hysteresis loops whose size depends on the cycling rate. Short-lived metastability results in relaxation hysteresis which, in the limit of very slow cycling, shrinks to the equilibrium curve. Stable hysteresis, however, does not decrease in size when the cycling velocity is diminishing (within the definition of time-independence for the underlying long-lived metastable states).

\subsection{Entropy Production}

The direction of rotation around a hysteresis loop is fixed by the entropy increase which accompanies directional (irreversible) changes of state. The entropy change $d S$ of such a reaction system is correlated with changes in the internal energy $\mathrm{d} U$, the work term $\mathrm{d} W$ and the chemical contribution $A \mathrm{~d} \xi$, through the Gibbs equation for chemical reactions (or phase changes) that proceed irreversibly at the (absolute) temperature $T^{[23]}$.

$T \mathrm{~d} S=\mathrm{d} U+\mathrm{d} W+A \mathrm{~d} \xi$

In the equations defining the affinity and the variation of the advancement of reaction, respectively

$A=-\sum_{j} v_{j} \mu_{j} ; \quad \mathrm{d} \xi=v_{j} \mathrm{~d} n_{j}$

$\mu_{j}$ denotes the chemical potential, $v_{j}$ the stoichiometric coefficient, and $n_{j}$ is the number of moles of component $j$ (or of a component in phase $j$ ).

In nonequilibrium thermodynamics, the entropy change of a reaction system is divided into the two terms $\mathrm{d}_{\mathrm{e}} S$ and $\mathrm{d}_{\mathrm{i}} S^{[24]}$. Thus

$\mathrm{d} S=\mathrm{d}_{\mathrm{e}} S+\mathrm{d}_{i} S$

The term $\mathrm{d}_{\mathrm{e}} S$ gives the contribution of the entropy which can be exchanged (as heat $\mathrm{d}_{e} Q$ ) with the environment, $\mathrm{d}_{\mathrm{e}} S=\mathrm{d}_{\mathrm{e}} Q / T$, whereas $\mathrm{d}_{\mathrm{i}} S$ represents the irreversible contribution created internally within the system. By definition $\mathrm{d}_{\mathrm{i}} S$ is positive, $\mathrm{d}_{\mathrm{i}} S \geq 0$. If we now utilize the first law, $\mathrm{d} U=\mathrm{d}_{\mathrm{e}} Q-\mathrm{d} W$ and insert Eq. (3) into Eq. (1), we obtain

$T \mathrm{~d}_{\mathrm{i}} S=A \cdot \mathrm{d} \xi$

This relationship states that there is entropy production $\left(\mathrm{d}_{\mathrm{i}} S>0\right)$ in a reaction system only if the reaction or phase change proceeds irreversibly (i.e. if $A>0$ ).

The entropy production accompanying a hysteresis cycle, $\Delta_{\mathrm{i}} S$, is derived by cyclic integration of Eq. (4):

$\Delta_{\mathrm{i}} S=\oint \mathrm{d}_{\mathrm{i}} S=\oint \frac{A}{T} \mathrm{~d} \xi$

As noted earlier, the intrinsic directionality of hysteresis offers the possibility of recording "information" (and of erasing the record by continuing the cycle). This information (=negentropy) is based on entropy production of the recording matrix. The cost of storing as well as of erasing the imprint is "paid for" by investing work. That entropy production means loss of useful work can be derived directly from Eq. (1). For a closed cycle we have (at constant $T$ )

$T \oint \mathrm{d} S=\oint \mathrm{d} U+\oint \mathrm{d} W+\oint A \mathrm{~d} \xi$

Since the state variables $S$ and $U$ depend only on the state of the system, (for a variation which returns to the original state) the cyclic integrals $\oint \mathrm{d} U$ and $\oint \mathrm{d} S$ equal zero. Thus Eq. (6) reduces to

$-\oint \mathrm{d} W=\oint A \mathrm{~d} \xi$ 
With Eq. (5) we obtain:

$\Delta_{\mathrm{i}} S=-\frac{1}{T} \oint \mathrm{d} W$

For $\Delta_{\mathrm{i}} S>0$, it follows that $\oint \mathrm{d} W<0 ;$ i.e., in order to close an irreversible cycle, work must be invested in the system.

\subsubsection{Isothermal-Isobaric Hysteresis}

The change in free energy $\mathrm{d} G$ accompanying a chemical reaction that proceeds irreversibly at constant temperature and at constant pressure $p$, is given by

$(\mathrm{d} G)_{p, T}=-A \mathrm{~d} \xi<0$

We now denote the product $-T\left(\Lambda_{\mathrm{j}} S\right)_{p, T}$ as $\left(\Delta_{\mathrm{i}} G\right)_{p, T^{\prime}}$, being the amount of free energy which is lost during an isothermal-isobaric hysteresis cycle.

$\left(\Delta_{\mathrm{i}} G\right)_{p . T}=-T\left(\Delta_{\mathrm{i}} S\right)_{p . r}$

This energy dissipation results from all processes $k$ which occur irreversibly. Comparing Eq. (5) and Eq. (10) we may write:

$\left(\Delta_{\mathrm{i}} G\right)_{p . r}=-\sum_{k} \int_{0}^{\xi_{k}} A_{k} \mathrm{~d} \xi_{k}$

In Eq. (11), $\xi$ is normalized and, being the relative extent of reaction or degree of transition, varies between $\xi=0$ and $\xi=1$.

\subsubsection{Calculation of $\Lambda_{i} G$}

Following the scheme of Figure 3, the calculation of $\Delta_{i} G$ may be performed in a general way. Using the symbols

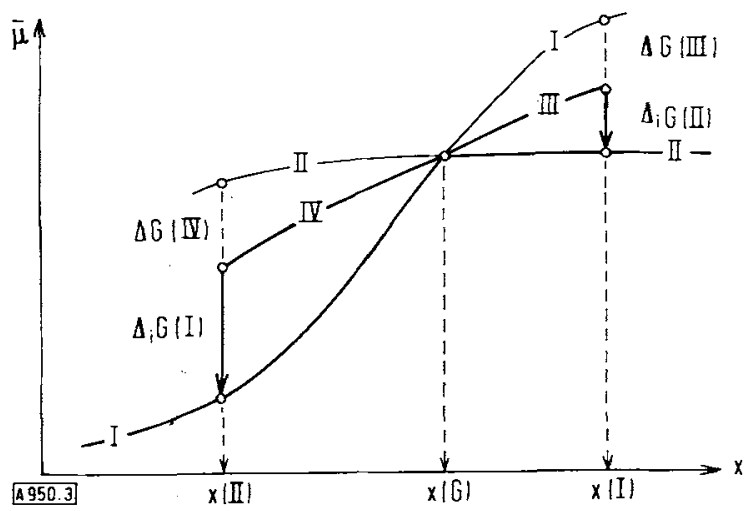

Fig. 3. Projection onto a $\mu-x$ plane of the mean chemical potentials $\bar{\mu}$, associated with the components or states. Cf. text and Fig. $2 \mathrm{a}$.

given in Figure $2 \mathrm{a}$ we may choose the states $I$ and II as reference states. The difference in free energy $\Delta G(\mathrm{II})$ between the states II and I at $x$ (II) is formally equal to the sum of the free energy changes $\Delta G$ (IV) accompanying the transition $\mathrm{II} \rightarrow \mathrm{IV}$ at $x(\mathrm{II})$ and the irreversible portion $\Delta_{\mathrm{i}} G(\mathrm{l})$.

$\Delta G(\mathrm{~J})=\Delta G(\mathrm{IV})+\Delta_{\mathrm{i}} G(\mathrm{I})$
At $x(I)$ we have correspondingly:

$\Delta G(\mathrm{I})=\Delta G(\mathrm{III})+\Delta_{i} G(\mathrm{II})$

Since $\Delta_{\mathrm{i}} G=\Delta_{\mathrm{i}} G(\mathrm{I})+\Delta_{\mathrm{i}} G(\mathrm{II})$, the total dissipation of free energy during a hysteresis cycle is given by

$\Delta_{\mathrm{i}} G=\Delta G(\mathrm{II})+\Delta G(\mathrm{I})-\Delta G(\mathrm{IV})-\Delta G(\mathrm{III})$

\subsubsection{Thermal (Isobaric) Hysteresis}

Provided that for thermal hysteresis the degree of transition $\xi$ can be determined as a function of temperature, we derive from Eq. (5) a relationship for the measurement of the entropy production $\left(\Delta_{\mathrm{i}} S\right)_{p}$.

$\left(\Delta_{i} S\right)_{p}=\oint\left[\sum_{k} \frac{A_{k}}{T}\left(\frac{\mathrm{d} \xi_{k}}{\mathrm{~d} T}\right)\right] \mathrm{d} T$

\subsection{Energy Dissipation of Potentiometric Hysteresis}

The hysteresis loops obtained in isothermal-isobaric potentiometric acid-base titrations are a direct measure of the energy dissipation and entropy production ${ }^{[25]}$. In a potentiometric hysteresis (cf., e.g., Fig. 4) the mean degree of protonation $\bar{\alpha}$ corresponds to the internal parameter $y$ and the $\mathrm{pH}$ value corresponds to $x$ (in the notation of Fig. 2). The degree of protonation is defined as the ratio between the number of moles of bound protons and the number of moles of protonation partners.

The usual condition of such an acid-base titration is the retention of constant ionic strength during the titration cycle. This can be achieved by using a neutral salt whose concentration is large as compared to the concentrations of the reactants. If in addition the acid anion and salt anion are identical, e.g. are $\mathrm{Cl}^{-}$ions, we may consider the chemical potential of the anions to be constant, and obtain, for instance, for the combination of $\mathrm{HCl}$ and $\mathrm{NaCl}$ : $\mathrm{d} \mu_{\mathrm{HCl}}=\mathrm{d} \mu_{\mathrm{H}^{+}}+\mathrm{d} \mu_{\mathrm{Cl}^{-}} \simeq \mathrm{d} \mu_{\mathrm{H}^{+}}$. Under this condition, the contribution of protonation to the change of the chemical potential of the (protonated) reactant $j$, is related (to a good approximation) to its degree of protonation $\alpha_{j}$ and the $\mathrm{pH}$ value by

$\left(\frac{\partial \mu_{j}}{\partial \mathrm{pH}}\right)_{n \neq n_{\mathrm{HC}}}=-2.3 R T x_{j}$

The notation $n \neq n_{\mathrm{HCl}}$ means constant $n$ except for $n_{\mathrm{HCl}}$ (cf. ${ }^{[8]}$ ).

The contribution of component $j$ to the change of the free energy $\Delta G_{k}$ of the $k$-th protonation reaction in an acid-base cycle can be derived by cyclic integration of Eq. (14).

$\Delta G_{k}=\oint\left(\frac{\partial \mu_{j}}{\partial \mathrm{pH}}\right)_{k} \mathrm{dpH}=-2.3 R T \oint \alpha_{j k} \mathrm{dpH}$

Since by definition

$\Delta G_{k}=-\int_{0}^{\xi_{k}} A_{k} \mathrm{~d} \check{\zeta}_{k} ; \quad\left(\Delta_{\mathrm{i}} G\right)_{p . T}=\sum_{k} \Delta G_{k}$ 

we obtain from Eq. (11) and with $\alpha=\sum_{j} \sum_{k} \alpha_{j k}$ for the total
dissipation of free energy

$\left(\Lambda_{i} G\right)_{p . T}=-2.3 R T \hat{\phi} \bar{\alpha} \mathrm{dpH}$

The integral $\oint \bar{\alpha} \mathrm{dpH}$ is geometrically the area of the potentiometric hysteresis loop. Thus $\left(\Delta_{i} G\right)_{p . T}$ and the entropy production

$\Delta_{\mathrm{i}} S=2.3 T \oint \bar{\alpha} \mathrm{dpH}$

can be experimentally determined.

Calculation of $\bar{\alpha}$. The number of bound protons is conveniently calculated from the difference in the amounts of acid or base which are necessary to bring the solution or suspension of the system to the same $\mathrm{pH}$ value as a "blank" solution. Since the investigation of hysteresis phenomena requires more than one acid-base cycle, more detailed relations (as usually needed) have to be used in order to calculate $\bar{\alpha}$. Such general equations have been given by Revzin, Neumann, and Katchalsky ${ }^{[7]}$.

\subsection{Protonation Energy of Polyelectrolyte Systems}

In those regions of the hysteresis in which protonation is reversible or apparently reversible (as in the metastable regions), the formalism of protonation equilibrium may be applied. For polyelectrolyte systems, the $\mathrm{pH}$ dependence of the degree of protonation $\alpha_{j}$ of the group $j$ is given by

$\log \frac{1-\alpha_{j}}{x_{j}}=\mathrm{pH}-\mathrm{p} K_{j}^{0}-\Delta \mathrm{p} K_{j}$

In Eq. (19), $\mathrm{p} K_{j}^{0}$ is the $\mathrm{p} K$ value of the single monomer residue and the polyelectrolyte contribution to the $\mathrm{p} K$ shift is defined by the relation

$\Delta \mathrm{p} K_{j}=\frac{z_{j} \cdot e \cdot \psi_{j}}{2.3 k T}$

In Eq. (20), $e$ is the (positive) elementary charge, $k$ is the Boltzmann constant, $\psi_{j}$ is the electrical potential at the site of protonation $j$, and $z_{j}$ is the charge number (with sign) of the groups which give rise to $\psi_{j}$. The value of $\psi_{j}$ depends on the geometry of the system, the mean degree of protonation and on the ionic strength of the medium.

The thermodynamic analysis of potentiometric hysteresis cycles often requires a knowledge of the free energy of protonation $\Lambda_{\mathrm{p}} G$. For the residue $j$, the standard value of this quantity is given by

$\Delta_{\mathrm{p}} G_{j}^{0}=-2.3 R T\left(\mathrm{p} K_{j}^{0}+\Delta \mathrm{p} K_{j}\right)$

Provided the $\mathrm{p} K$ value of the monomer residue is known, $\Delta_{\mathrm{p}} G_{j}$ can be determined by calculating $\Delta \mathrm{p} K_{j}$ (cf. ${ }^{[26]}$ )

\subsection{Aim of Thermodynamic Hysteresis Analysis}

The thermodynamic relations discussed in Section 2 provide the general framework for the analysis of hysteresis phenomena in chemical reactions or phase changes. The central task of such an analysis comprises the identification of those processes which occur irreversibly. For this purpose a comparison of measured and calculated values for $\Delta_{\mathrm{i}} G$ may be useful.

Especially in polyelectrolyte systems, the thermodynamic analysis of temperature and ionic strength dependences may give hints as to the nature of the energy barrier which causes the metastable states and forces the system to hysteretically circumvent this barrier.

From a comparison of the Eqs. (8) and (10) we see that $\left(\Delta_{\mathrm{i}} G\right)_{p . T}$ is a measure of the energy investment for an isothermal-isobaric hysteresis cycle. Therefore the thermodynamic analysis leads in principle to the determination of the free energies which must be invested in the system in order to re-establish the metastable states. The role of metastable states in triggering directed processes, at low expenditures of energy, is discussed in Sections 7 and 8 .

\section{Hysteresis in Nucleic Acids}

\subsection{Synthetic Polynucleotides}

Especially pronounced hysteresis phenomena have been observed in potentiometric and spectrophotometric titrations of complexes composed of homogeneous synthetic nucleic acids. As a representative example, the result of a potentiometric acid-base titration of the polynucleotide complex poly(A).2 poly(U) is shown in Figure 4 (cf. ${ }^{[8]}$ ).

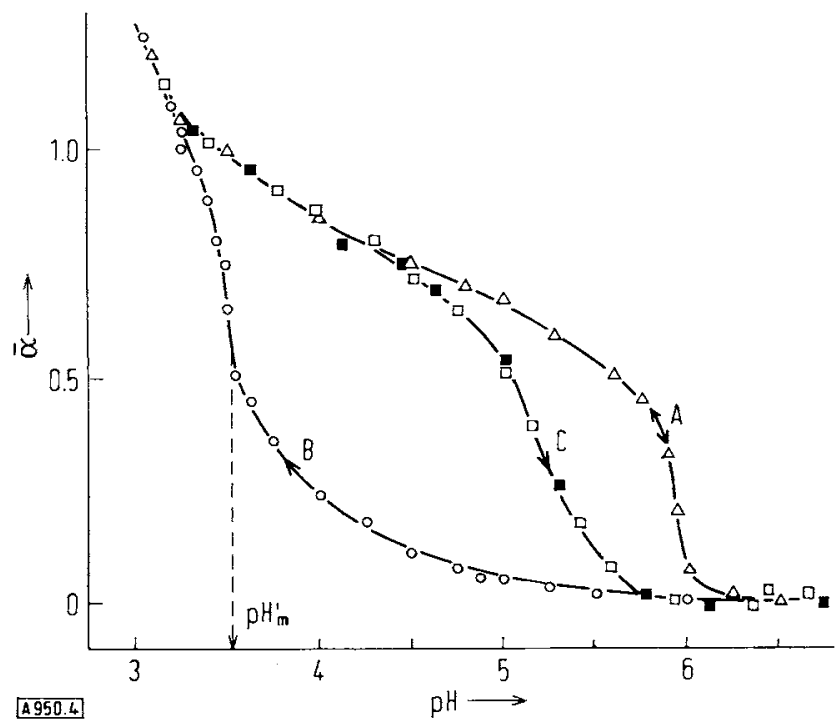

Fig. 4. Potentiometric titrations (degree of protonation $\bar{\alpha}$ as a function of $\mathrm{pH}$ ) at $20^{\circ} \mathrm{C}$ : a) $0.1 \mathrm{M} \mathrm{NaCl}$ solution of poly(A) and poly(U) mixed in the molar ratio of the polymers $1: 2 ; 0$ : acid titration (curve $B$ ): $\circ$, subsequent base titration (curve C). b) $0.1 \mathrm{M} \mathrm{NaCl}$ solution of poly(A); $\Delta$, (curve A). Polymer concentrations at $\mathrm{pH} 7:$ a) $c=3.24 \times 10^{-4} \mathrm{M}$ (A. $2 \mathrm{U})$; b) $c=3.2 \times 10^{-4} \mathrm{M}(\mathrm{A})$ 
The three-stranded (A - 2 U) complex is formed when polyriboadenylate, poly(A), and polyribouridylate, poly(U), are mixed at neutral $\mathrm{pH}$ and sufficiently high ionic strength in the molar ratio of the polymers $1: 2^{[27]}$. The doublestranded complex poly(A) p poly(U) may be obtained upon mixing the single strands in the molar ratio $1: 1^{[28]}$. In these helical multistranded complexes the heterocyclic bases adenine and uracil are specifically associated by way of $\mathrm{H}$-bonds and regularly stacked upon each other. Due to the cooperatively stabilized base stacking such systems may be considered as "unidimensional" crystals. (Because of their polyelectrolyte nature the stability of multistranded polynucleotide complexes is strongly dependent on the ionic strength of the medium.)

The first indications of metastable forms in the A-U system are seen in the results of Warner and Breslow: the (A.U) complex, apparently stable at $\mathrm{pH} 5.5$ with respect to the single strands, is formed only to a small extent if the polymers are first separately brought to $\mathrm{pH} 5.5$ and then mixed together ${ }^{28]}$. Similar results were obtained by Steiner and Beers ${ }^{[29]}$. Later, Cox discovered that the size of the hysteresis loops observed in spectrophotometric titrations of $(A \cdot U)$ complexes are dependent on temperature ${ }^{[30]}$. Poly(A), too, shows dependence on previous history[29.31]. The partially protonated double helix poly(A) - poly(A) that is formed at acidic $\mathrm{pH}$ values, has a tendency to aggregate with increasing proton concentration. At a given $\mathrm{pH}$, the extent of this aggregation depends on the ionic strength. Furthermore, the actual state of the system is determined by the sequence in which the parameters $\mathrm{pH}$ and ionic strength are changed. The aggregation of poly(A) occurs relatively slowly at $\mathrm{pH}$ values which are not too low, so that this aggregation does not seriously disturb experimental studies on the acid-base hysteresis in the A-U-system. (Aggregation of larger extent can be checked by the measurement of sedimentation coefficients.)

\subsubsection{The $(A \cdot 2 U)$ Model System}

The first thermodynamic analysis of molecular hysteresis was performed by Katchalsky, Oplatka, and Litan using Cox's results for the A-U system ${ }^{[25]}$. In contradistinction to the original interpretation, however, neither the $(A \cdot U)$ double helix nor the $(A \cdot A)$ complex can here develop long-lived metastability, but only the $(A \cdot 2 U)$ triple strand $^{[8]}$.

The hysteresis in the acid-base titration of the A-U system accompanies the "transcrystallization" reaction between the $(\mathrm{A} \cdot 2 \mathrm{U})$ triple helix and the $(\mathrm{A} \cdot \mathrm{A})$ double strand. The overall reaction may be written (in terms of the reactive chain residues or segments) as

$2(A \cdot 2 U) \mathrm{H}^{+}(A \cdot A)+4(U)$

In the pH range 3 to 7 , the measured quantity $\bar{\alpha}$ (as shown in Fig. 4) reflects protons bound to (A) residues. In the course of the acid titration the $(\mathrm{A} \cdot 2 \mathrm{U})$ complex does not directly transform to the protonated $(A \cdot A)$ helix, but is first (partially) protonated. (This conclusion is supported by the similarity of the sedimentation coefficient distributions between $\mathrm{pH} 7$ and $\mathrm{pH}$ 4.) Thus, the measured quantity $\bar{\alpha}$ is a sum of two contributions, one from protons bound to the $(\mathrm{A} \cdot \mathrm{A})$ double helix (degree of protonation $\alpha_{2}$ ) and the other from protons bound to the triple helix (degree of protonation $\alpha_{3}$ ). If $\xi$ denotes the extent of the conformational change described in Eq. (22), we obtain for each $\mathrm{pH}$ value:

$\bar{\alpha}=\xi \alpha_{2}+(1-\xi) \alpha_{3}$

The value of $\alpha_{2}$ can be measured independently by titration of poly $(A)$, whereas $\alpha_{3}$ may be calculated using a relation analogous to Eq. (19); see Eq. (35).

In a similar way UV-spectroscopic titration data can be used to determine the $\mathrm{pH}$-dependence of $\xi$. Figure 5 shows

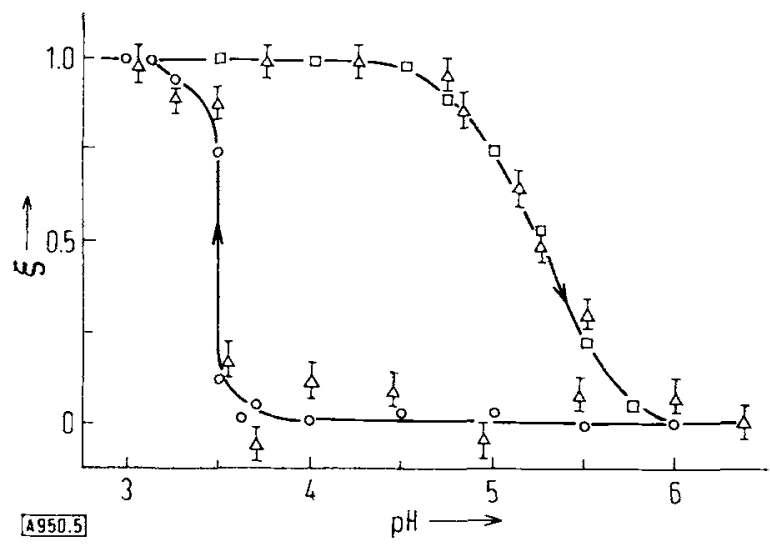

Fig. 5. Extent of reaction $\xi$ as a function of $\mathrm{pH}$. Experimental conditions as in Figure 4. 0 , 0 : data of potentiometric and $\Delta$ : data of spectrophotometric titrations. Cf. [8]

that the same $\xi(\mathrm{pH})$ is obtained irrespective of whether the quantity measured is $\bar{\alpha}$ or UV absorbance ${ }^{[8]}$. Furthermore, it is clearly seen that, along the acid branch, $(\mathrm{A} \cdot \mathrm{A})$ formation occurs only at $\mathrm{pH}$ values near to $\mathrm{pH} 3.5$. The transition on the acid branch is abrupt, whereas the smooth base branch points to an equilibrium transition.

a) Molar state variables. The measured (apparent) state variables of high molecular weight polynucleotides are independent of polymer concentration $c_{j}{ }^{[18.32]}$. Therefore the polymer activity coefficients may be taken as constant and thus can be incorporated into the standard potentials $\mu_{j}^{0}$. Thus

$\Delta G=\sum_{j} v_{j} \mu_{j}=\sum_{j} v_{j} \mu_{j}^{0}+R T \sum_{j} v_{j} \ln c_{j}$

For unit stoichiometric conversion, i.e. all $c_{j}=1 \mathrm{~mol} / \mathrm{l}$, it follows that

$\Delta G^{0}=\sum_{j} v_{j} \mu_{j}^{0}$

and with Eq. (16) we obtain:

$\left(\Delta_{i} G\right)^{0}=\sum_{k}\left(\sum_{j} v_{j k} \mu_{j}^{0}\right) \xi_{k}$

If we relate the state parameters to the number of moles of (A) chain segments, the measured $\bar{\alpha}(\mathrm{pH})$ curve of Figure 4 directly yields the free energy loss, per mole of (A), for one isothermal-isobaric hysteresis cycle:

$\left(\Delta_{i} G\right)_{p . T}^{0}=\sum_{\hat{k}} \Delta G_{k}^{0} \xi_{k}=-2.3 R T \oint \bar{\alpha} \mathrm{dpH}$ 
b) Calculations of $\left(\Delta_{\mathrm{i}} G\right)^{\circ}$. As mentioned above there are indications that the base branch of the hysteresis in the A-U system is the equilibrium curve ${ }^{[8.33]}$. Consequently our model system appears to have only one region of metastability. In this region the partial protonation of the triple helix represents a metastable pre-equilibrium. This equilibrium comprises the opening of $(\mathrm{A} \cdot 2 \mathrm{U})$ segments

and the protonation of open (A) segments, according to (A) $+\mathrm{H}^{+}=\left(\mathrm{AH}^{+}\right)$.

The protonation equilibrium corresponds to state IV in Figure $2 \mathrm{a}$. Thus, state II represents the $(A \cdot 2 \mathrm{U})$ triple helix, whereas state I represents the $(\mathrm{A} \cdot \mathrm{A})$ double helix. If we now choose the chemical potentials of the separated single strands as reference, the calculation of $\left(\Delta_{\mathrm{i}} G\right)^{0}$ becomes particularly simple. For our model system the general case as represented in Figure 3 is reduced to the scheme shown in Figure $6^{[8]}$.

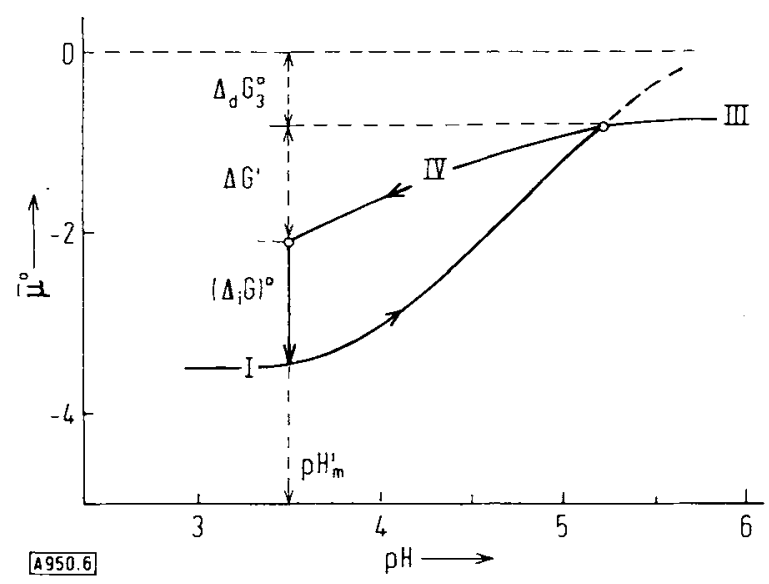

Fig. 6. Projection onto a potential-pH plane of the mean molar standard potentials $\bar{\mu}^{\mathrm{o}}$ associated with the nucleotide segments; $\Delta G^{\prime}=\alpha_{3}^{\prime}$. $\left(\Delta_{\mathrm{d}} G_{3}^{0}+\Delta_{\mathrm{p}} G_{3}^{0}\right)$, see Eq. (29).

By use of Eqs. (25) and (26) we can derive an expression for $\left(\Delta_{\mathrm{i}} G\right)^{0}$ which corresponds to Eq. (12).

$\left(\Delta_{\mathrm{i}} G\right)^{0}=\Delta_{\mathrm{b}} G_{2}^{0}+\Delta_{\mathrm{d}} G_{3}^{0}+\alpha_{3}^{\prime}\left(\Delta_{\mathrm{d}} G_{3}^{0}+\Delta_{\mathrm{p}} G_{3}^{0}\right)$

In Eq. (29), $\Delta_{b} G_{2}^{0}$ is the change in the free energy accompanying the reaction $(\mathrm{A})+\mathrm{H}^{+}=\frac{1}{2}\left(\mathrm{AH}^{+} \cdot \mathrm{AH}^{+}\right)$at $\mathrm{pH}_{\mathrm{m}}^{\prime}$ (corresponding to $x(\mathrm{II})$ in Fig. 3), $\Delta_{\mathrm{d}} G_{3}^{0}$ is the free energy of the helix-coil transition (Eq. (28)), $\Delta_{\mathrm{p}} G_{3}^{0}$ is the free energy change for the protonation of (A) segments in the triple helix (and may be calculated with Eq. (21)), and $\alpha_{3}^{\prime}$ is the extent of protonation at $\mathrm{pH}_{\mathrm{m}}^{\prime}$.

It has been shown that, based on the assumptions underlying Eq. (29), the calculation and measurement of $\left(\Delta_{\mathrm{i}} G\right)^{0}$ are in agreement ${ }^{t 8 !}$. Under the experimental conditions of Figure 4 we find that

$\left(\Delta_{i} G\right)_{p, T}^{0}=-1.4( \pm 0.1) \mathrm{kcal} / \mathrm{mol}(\mathrm{A})$

$\left(\Delta_{i} S\right)_{p, T}^{0}=4.9( \pm 0.1) \mathrm{cal} / \mathrm{mol}(\mathrm{A}) \mathrm{deg}$
The agreement of the measured and the calculated $\Delta_{i} G$ value supports the interpretation of the hysteresis behavior, which was the basis of the calculation leading to Eq. (29). Accordingly, the hysteresis in our model system implies that a metastable protonation equilibrium exists as an intermediary during the acid titration.

It may be added that the energy loss in this hysteresis system is a rather small energy investment as compared to the average energy of stabilization of a chemical bond ( $\approx 50 \mathrm{kcal} / \mathrm{mol}$ ).

\subsection{2. $(\mathrm{I} \cdot \mathrm{C})$ and $(\mathrm{dC} \cdot \mathrm{dG})$ Complexes}

It appears that three-stranded polyribonucleotide complexes (as opposed to double-stranded helices) tend to developlong-lived metastabilities. Polymer associates similar to the (A.2U) complex occur also in complexes of homopolymers of inosinic(I)-, cytidylic(C)-, and guanylic (G) acids. Guschlbauer and co-workers found that in the system poly $(\mathrm{I}) \cdot \operatorname{poly}(\mathrm{C})$ there are two hysteresis regions which are separated from each other by an equilibrium range ${ }^{[34]}$. A partially protonated triple strand of type (I - C C C) can be identified as an intermediate in the course of the $(\mathrm{I} \cdot \mathrm{C})$ conversion $^{\left[{ }^{35}\right]}$. (This is similar to the $(\mathrm{A} \cdot 2 \mathrm{U}$ ) intermediate in the $(\mathrm{A} \cdot \mathrm{U})$ conversion to $(\mathrm{A} \cdot \mathrm{A})$ base pairs $^{[8]}$.)

Extended regions of metastability are observed in polydeoxyribonucleotides. Thus, the acid-base hysteresis in poly $(\mathrm{dG}) \cdot$ poly $(\mathrm{dC})$ covers about $6 \mathrm{pH}$ units (between $\mathrm{pH}$ 2 and 8 ). This type of base pairing exhibits metastability in the physiologically relevant neutral $\mathrm{pH}$ range. Since it is known that rather long $(\mathrm{dG} \cdot \mathrm{dC})$ stretches exist in DNA, we have here a possibility for a regulatory mechanism which could be specifically based on metastabilities in clustered $(\mathrm{dC} \cdot \mathrm{dG})$ base pairs ${ }^{[34]}$.

The energetics of such a mechanism could be estimated by a thermodynamic analysis of the hysteretic behavior of the $(\mathrm{dG} \cdot \mathrm{dC})$ complex. In principle such an analysis can be performed in a similar way as in the $(A \cdot 2 U)$ system.

\subsubsection{Thermal Hysteresis in Biopolymers}

The UV-absorbance of some complexes composed of poly(dA) and polymers of substituted uridylic acids changes along a hysteresis loop during a heating-cooling cycle ${ }^{[36]}$. Similar thermal hysteresis has been observed in thio derivatives of the copolymer poly $(\mathrm{dA}-\mathrm{T})$ in solutions of high salt concentration $(2 \mathrm{M} \mathrm{NaCl})^{[37]}$. The hysteresis in this copolymer accompanies conformational changes between different helix types.

The capability of developing metastable states is apparently not restricted to complexes composed of several polyelectrolyte strands. For instance, the complex poly(A) - poly(-Ivinyluracil) in which only poly(A) is an electrolyte, shows thermal hysteresis within a relatively broad temperature range $^{[38]}$.

There is a series of examples where hysteresis is due to unspecific aggregation and disaggregation of molecules. This category probably includes all the thermal hysteresis loops which have been found for a series of synthetic nucleic acids in water-methanol mixtures at lower temperatures $^{[39]}$. 
Since optical parameters like UV absorbance at certain wavelengths are a measure of the degree of transition $\xi$ of conformational changes, we may use Eq. (13) as a starting point for a thermodynamic description of thermal hysteresis in those biopolymers in which structural changes can be followed by optical methods.

\section{2. rRNA Hysteresis}

Long-lived metastabilities can be developed not only by long polynucleotides but also by shorter multistranded structured regions in nucleic acids. An example of such a system is the hysteresis of rRNA (cf., e.g., ${ }^{[40]}$ ). Recent studies have shown that the rRNA hysteresis is clearly an intramolecular phenomenon ${ }^{[7]}$. The formation of metastable states in this biopolymer is linked with the presence of $\mathrm{Mg}^{2+}$ ions. Furthermore, the metastabilities occur only along the base branch of the boundary loop and along the scanning curves, whereas the acid branch represents the equilibrium curve. From the high thermal stability of the metastable forms and from the analysis of the UV spectrum as a function of $\mathrm{pH}$, it was concluded that structured domains comprising mainly protonated $G \cdot G$ base pairs are the cause of the long-lived metastabilities in rRNA.

Questions concerning the possible role of metastable states in rRNA structures and in the regulation of ribosomal processes are the subject of further investigations ${ }^{[7]}$.

\section{Hysteresis in Proteins and Membranes}

Hysteresis is also observed for structural changes in "globular" polyelectrolyte systems such as proteins, and lipoprotein and lipid vesicles. The hysteresis obtained in polyglutamic acid is considered representative for metastability in polypeptides. In this case the acid-base hysteresis has been interpreted in terms of specific aggregation-disaggregation processes $^{[41]}$

For proteins, acid-base titrations are frequently used in order to determine type and number of buried groups ${ }^{[42]}$. However, the metastability indicated in the titration curves has rarely been thermodynamically analyzed. It should be stressed that the dissipation of free energy, $\Lambda_{i} G$, of such systems represents a direct measure of the free energy stabilizing buried groups within the biopolymer.

An example of functional hysteresis in proteins has been discovered in the halophilic enzyme malate-dehydrogenase isolated from halobacteria of the Dead Sea ${ }^{[4]}$. The change of the catalytic activity of this enzyme with decreasing and increasing salt concentration follows a hysteresis loop (see Fig. 7).

Besides the hysteresis in nerve membranes ${ }^{[14.15]}$, metastabilities are also observed in suspensions of lipid vesicles. The thermal hysteresis found for such a spherical membrane system is bound to the presence of di- or trivalent ions or acetylcholine ${ }^{\{20]}$.

The acid-base titration of membrane fragments of the cell envelopes prepared from Dead Sea halobacteria yields a hysteretic proton binding curve (see Fig. 8). The size

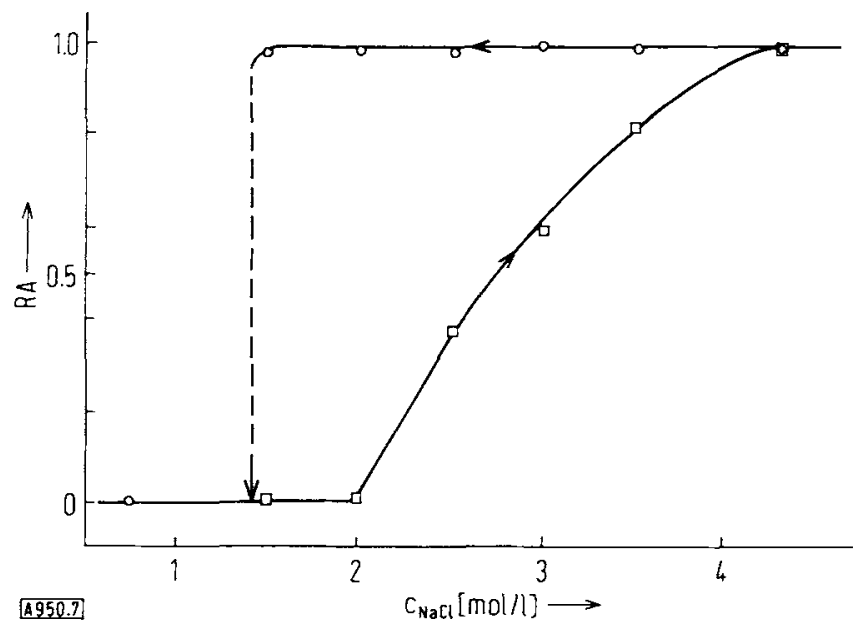

Fig. 7. Relative enzymatic activity, RA, of the haloenzyme malate-dehydrogenase at $30^{\circ} \mathrm{C} ; O$, values measured at decreasing and $\square$, values measured at increasing $\mathrm{NaCl}$ concentration $C_{\mathrm{NaCl}}[43]$

of this membrane hysteresis is dependent on the salt concentration; at a $\mathrm{NaCl}$ content smaller than $1 \mathrm{M}$, the hysteresis shrinks to give the equilibrium curve. If, at constant $\mathrm{pH}$, the salt concentration is decreased and increased in a cyclic mode, one also obtains a hysteresis loop ${ }^{[4]}$.

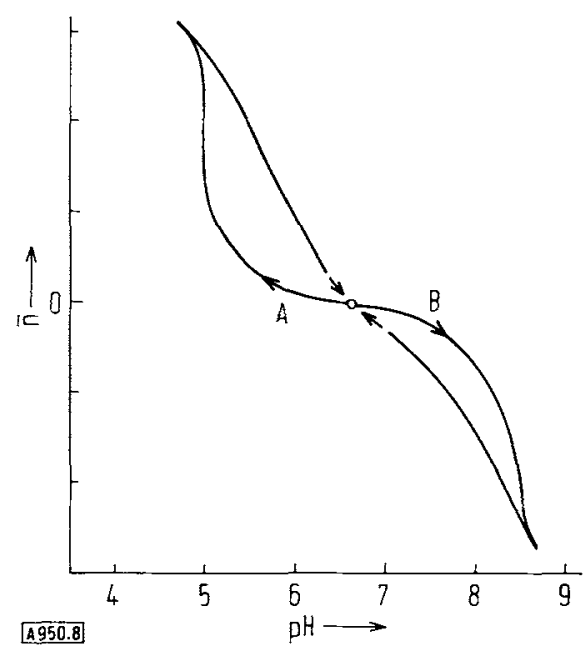

Fig. 8. Proton binding curve of suspended membrane fragments prepared from cell envelopes of Dead Sea halobacteria; $\bar{n}$, mean number of protons bound (in arbitrary units) as a function of $\mathrm{pH}$. A, acid-base cycle in the acid $\mathrm{pH}$ range; $\mathrm{B}$, base-acid cycle in the alkaline $\mathrm{pH}$ range; $20^{\circ} \mathrm{C}$, $3 \mathrm{M} \mathrm{NaCl}$

It is seen from Figure 8 that the nonequilibrium transitions indicated in the acid-base hysteresis occur in $\mathrm{pH}$ ranges which include the $\mathrm{p} K$ values associated with primary amino- and carboxyl groups of membrane proteins (at $3 \mathrm{M} \mathrm{NaCl}$ at about $\mathrm{pH} 8.5$ and $\mathrm{pH} 5$ ). The hysteresis in these membrane fragments is probably due to metastabilities in the "ion pairs" of electrostatically associated side groups. It appears that electrostatic interactions also contribute to the existence of hysteresis in the activity of our haloenzyme. In this protein, hysteresis derives from long-lived metastable states in the association-dissociation reaction between the dimer subunits of the tetrameric enzyme complex ${ }^{[43]}$. 
The cybernetic significance of hysteresis in membranes and catalytically active proteins will be discussed in Section 8.

\section{Molecular Field Theory of Hysteresis}

A general thermodynamic description of hysteresis cannot, of course, answer the question of the physical origin of this nonequilibrium phenomenon.

In the molecular hysteresis of nucleic acid complexes, longlived metastability appears to be due to the cooperativity of base stacking and to the electrostatic interactions between the polyanions. Indeed, the first attempts at a molecular interpretation of the hysteresis in the A-U system include the assumption of an electrostatic nucleation barrier to the formation of protonated $(\mathrm{A} \cdot \mathrm{A})$ sequences ${ }^{[25,8]}$. In this approach, however, only a qualitative interpretation has been given.

Recently, a molecular field theory of hysteresis has been developed ${ }^{[45]}$. According to this approach, a large energy barrier prevents opening of (A.2U) segments within the partially protonated triple helix. (The increased protonation in the acid titration compensates a part of the electrostatic repulsion energy arising from the phosphate residues of opposite strands, such that further opening of $(\mathrm{A} \cdot 2 \mathrm{U})$ sequences is more difficult before a critical $\mathrm{pH}$ is reached.) We may formally write the mean free energy $\bar{G}$ for the stabilization of an associate such as the (A.2U) segment as a sum of two terms:

$\bar{G}=-\bar{A}+\bar{R}$

The term $\bar{A}$ comprises all attraction contributions, primarily short range forces such as van der Waals- and hydrophobic interactions. The second term, $\bar{R}$, accounts exclusively for (long range) electrostatic repulsion forces between charged groups. We may further specify our "molecular field" model for the triple helix by defining the completely separated (non-interacting) single strands as a reference state for which $\bar{R}=0$. (Thus $\bar{R}$ is the relative mean free energy of the dissociated nucleotide residues in the triple helix.)

In our model the energy barrier for the separation of associated chain residues arises from the different manner in which $\bar{A}$ and $\bar{R}$ depend on the average distance, $\bar{d}$, between the opposite association partners. We may assume that $\bar{A}$ is zero for $\bar{d}>d_{0}$, where $d_{0}$ is the distance of maximum attraction. In the simple case of Coulomb repulsion, $\bar{R}$ is proportional to $1 / \bar{d}$. Figure 9 schematically shows these correlations of $\bar{R}$ and $\bar{A}$ with $\bar{d}$.

Suppose now that the experimental conditions are such that only a few base residues within the cooperatively stabilized organization of the stacked $(\mathrm{A} \cdot 2 \mathrm{U})$ triple helix could dissociate. The dissociated chain residues could not separate very far because their closed neighbors prevent them from moving apart from each other. A small distance between dissociated residues, however, means a high value of the $\bar{R}$ term. Thus dissociation of only a few associates would create an energetically unfavorable state, i.e. dissociation encounters a nucleation barrier.

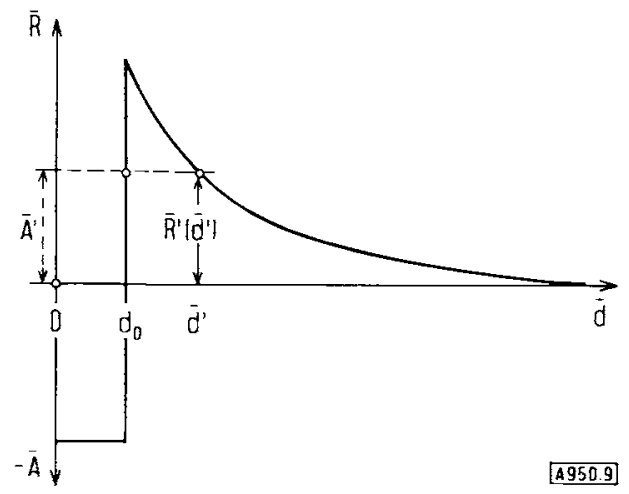

Fig. 9. Mean free energies $\bar{A}$ and $\bar{R}$ as a function of the mean distance $d$ between the association partners in polyelectrolyte structures; see text

This barrier can be decreased if $\bar{d}$ is increased. Such a decrease may be achieved by an increase in $\bar{A}$ (for instance, by a change in $\mathrm{pH}$ ) thereby decreasing $\bar{R}(\bar{d})$. The thermodynamic condition for the onset of separation is given by

$\bar{G}(\bar{d}) \geq 0$

If separation occurs at $\bar{d}=\bar{d}$, we have at this point $\bar{A}^{\prime}=\bar{R}\left(\overline{d^{\prime}}\right)$; see Fig. 9. Since now, however, $\bar{G}\left(\overline{d^{\prime}}\right)=\bar{A}^{\prime}>0$, the dissociation of the entire assembly and the separation of the strands proceed irreversibly. All states for which $0<\bar{A} \leq \bar{A}^{\prime}$ thus are metastable.

Since $\bar{R}$ depends on $\bar{d}$, it is obvious that $\bar{R}(d)$ is a function of the mean fraction, $\bar{f}$, of closed (A.2U) segments within a triple helix. We assume that this dependence is linear. Denoting the proportionality factor of this approximation by $B$, we may write

$\bar{R}=B \cdot \bar{f}$

It is easily seen that the term $\bar{R}$ has its maximum value if $\bar{f}=1$, while $\bar{R}=0$ for $\bar{f}=0$.

Since any chain segment in the triple helix is subject to the long-range electrostatic forces (or to the molecular field) of all segments, we may call Eq. (31) the molecular field approximation for our model.

In the range of experimental conditions, in which $\bar{G}$ depends on $\bar{d}$ and thereby on $f$, we may calculate the total free energy $G_{\mathrm{tot}}$ as a function of $f$ by the following relation:

$G_{\mathrm{ler}}=-\bar{A} \cdot f+(1-f) \cdot B \cdot f+R T[f \ln f+(1-f) \ln (1-f)]$.

It can be shown that for $B>2 R T$, the state function $G_{\text {tor }}$ has two minimum values, one being associated with the metastable state and the other with the thermodynamic equilibrium state. Such an energy diagram containing two minima has already been used by Borelius in order to interpret long-lived metastability and hysteresis ${ }^{[2]}$. 
An important result of the theory is a condition for the existence of thermodynamically metastable states:

$B>2 R T$

For our (A.2U) model system, the inequality (32) holds for lower temperatures $\left(<50^{\circ} \mathrm{C}\right)$, whereas for the $(A \cdot U)$ double helix $B<2 R T$. At the Curie temperatur $T_{c}$, we have $B=2 R T_{\mathrm{c}}$; that is, for $T \geq T_{\mathrm{c}}$, the hysteresis disappears and one obtains only the equilibrium curve ${ }^{[8]}$.

The hysteresis loops obtained in membranes and protein complexes of high surface charge densities may be molecularly interpreted with a molecular field approach in a manner similar to the polynucleotide systems.

\section{Hysteresis as an Analytical Method}

The extreme longevity of the metastabilities in some biopolymers and membrane structures permits the use of hysteresis as an analytical method for the investigation of distributions of structural subunits. As already mentioned, the occurrence of scanning curves implies domain structures. The analysis of these scanning curves can, in principle, reveal the distribution function of the domains and their characteristic transition points. Once this function is known, the scanning behavior can be predicted $^{[46,47]}$.

Such an analysis is based on the Preisach model ${ }^{[48]}$ which underlies Everett's domain theory. In the simple model of independent domains, the domain distribution function $\phi(x)$ can be determined in a relatively simple way from a limited number of scanning curves. This requires, however, that the mean extent $\xi_{\mathrm{i}}$ of the irreversible changes (i.e. the fraction of transformed domains) be measured as a function of the external parameter $x$. Let us attribute to each domain a lower transition point $x_{1}$ and an upper one $x_{u}$ (corresponding to $x(\mathrm{II})$ and $x(\mathrm{I})$ in Fig. 2). From the relationship

$\phi(x)=-\frac{\partial^{2} \xi}{\partial x_{\mathrm{u}} \partial x_{1}}$

the function $\phi(x)$ can be derived using experimental $\xi_{i}(x)$ values $^{[46]}$.

By assigning a lower and an upper $x$-limit for each scanning step we may describe scanning processes in a simple way. Thus, the paths by which a point of the hysteresis can be reached are represented by a domain complexion ${ }^{[46]}$. This function describes the path-dependent history of a domain system in a manner analogous to the after-effect function introduced in 1876 by Boltzmann for the description of elastic after-effects depending on the

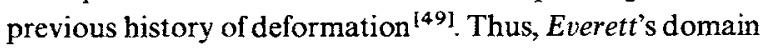
complexion is a type of memory function, and the domain distribution function describes the "memory capacity" of a multidomain system.

In order to check whether a system of independent domains is present, one may use a key theorem of Everett's theory. For independent domains the areas of $\xi_{\mathrm{i}}(x)$ scanning loops between the same $x$-limits have to be congruent.
If the $\xi_{\mathrm{i}}$ values cannot be evaluated, we may use a modification of this theorem due to Enderby ${ }^{[47]}$. In this case the measured scanning loops (e.g., $\bar{\alpha}(\mathrm{pH})$ curves) between the same $x_{1}$ - and $x_{u}$ values are compared. In a system in which the state of a domain does not depend on the states of the other domains, the intercepts at the same $x$ value between each scanning loop are equal.

If we utilize this theorem for the scanning loops in an $(\mathrm{A} \cdot 2 \mathrm{U})$ system with a relatively broad molecular weight distribution, we obtain the picture represented in Figure 10. It is seen that the "loop intercepts" $\Delta \bar{\alpha}(\mathrm{pH})$ are equal to within $10 \%$ accuracy. Thus the $(\mathrm{A} \cdot 2 \mathrm{U})$ complexes apparently behave independently.

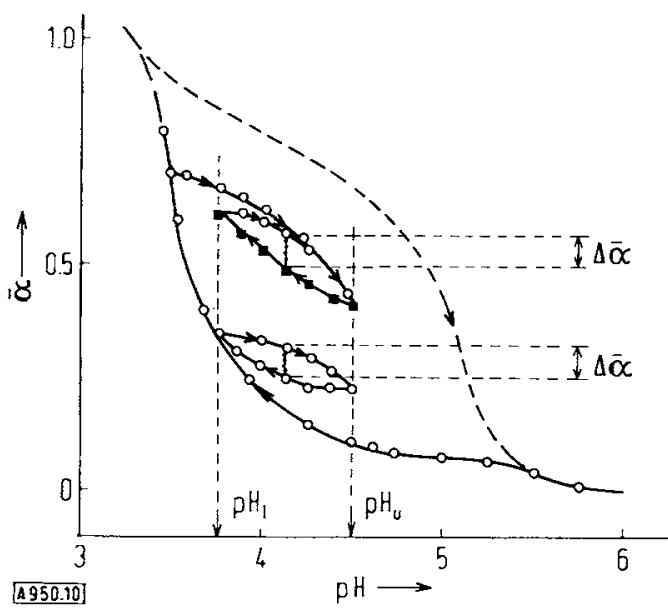

Fig. 10. Scanning loops in an (A-2U) system of broad molecular weight distribution; $\mathrm{pH}_{1}$ is the lower and $\mathrm{pH}_{u}$ is the upper $\mathrm{pH}$ limit of the loops. Comparison of the differences $\Delta \bar{\alpha}$ between the loops at the same pHi value; cl. Figure 4 and see text.

Assuming that $(\mathrm{A} \cdot 2 \mathrm{U})$ complexes of different molecular weight have different stabilities, the differentiation of the hysteresis boundary curve should yield the relative distribution of these stabilities and the relative amount of triple helices of different molecular weights along the $\mathrm{pH}$ scale. Thus, the analysis of the scanning behavior is in principle a method of determining the distribution of molecular weights in biopolymer mixtures.

\section{Conformational Changes Induced by Electric Impulses in Polyelectrolyte Systems}

Acid-base titrations are a standard technique for studying the thermodynamics of structural changes in ionizable systems. When the titrations are performed at different ionic strengths, it is possible to determine the electrostatic contributions to the energetics of structural changes. Such electrostatic contributions are particularly large in polyelectrolyte systems.

Eqs. (19) and (20) show that, for homogeneous polyelectrolyte systems:

$\log \frac{1-\alpha}{\alpha}=\mathrm{pH}-\mathrm{p} K^{0}-\frac{z e \psi}{2.3 k T}$

In this relationship we see that $\alpha$ may be changed by a variation in $\mathrm{pH}$ or by a change of the electrical potential 
term $\psi$. In general, $\psi$ is determined by the intramolecular field. This inner field may be influenced by the degree of ionization (protonation) and by the ionic strength of the medium, but also by external electric fields to which the system may be exposed.

In our hysteresis model system, the protonation pre-equilibrium of the $(\mathrm{A} \cdot 2 \mathrm{U})$ complex in the $\mathrm{pH}$-range above the irreversible transition at $\mathrm{pH}_{\mathrm{m}}^{\prime}$ may be described by the following relation:

$\log \frac{1-\alpha_{3}}{\alpha_{3}}=\mathrm{pH}-\mathrm{p} K_{\mathrm{AMP}}^{0}+\frac{e \psi}{2.3 k T}+\frac{\Delta_{\mathrm{d}} G_{3}}{2.3 k T}$

In Eq. (35), $\mathrm{p} K_{\mathrm{AMP}}^{0}$ is the $\mathrm{p} K$ value of adenosine monophosphate and $\Delta_{d} G_{3}$ corresponds to the $\mathrm{p} K$ shift due to the fraction of the (A) segments which dissociate from $(A \cdot 2 \mathrm{U})$ segments during protonation (cf. Eq. (28)). ( $\psi$ is negative, since it arises primarily from the negatively charged phosphate residues.) At $\alpha=\alpha_{3}^{\prime}$ corresponding to $\mathrm{pH}=\mathrm{pH}_{\mathrm{m}}^{\prime}$, irreversible formation of $(A \cdot A)$ sequences occurs.

A change in $\psi$ which leads to $\alpha>\alpha_{3}^{\prime}$ may be achieved at constant $\mathrm{pH}$ by a change in the ionic strength. Indeed, the irreversible formation of the $(\mathrm{A} \cdot \mathrm{A})$ double helix may be triggered by decreasing the salt concentration in a solution of metastable (A - 2U) triple strands ${ }^{[33]}$. Recently, we have found that electric impulses (of an initial intensity of about $20 \mathrm{kV} / \mathrm{cm}$ and decaying exponentially with a relaxation time of about $10 \mu \mathrm{s}$ ) directly induce this conformational transition ${ }^{[50]}$.

A polarization mechanism has been proposed to explain the electric induction of such conformational changes. In accordance with this idea, the external electric field shifts the ionic atmosphere of the polyelectrolytic $(\mathrm{A} \cdot 2 \mathrm{U})$ complex and thereby induces a dipole moment. At the negative pole of the induced macro-dipole, the screening by the ion cloud of the negative phosphate residues is reduced. This, in turn, causes repulsion between the ends of the polyanions and leads finally to the unwinding of the triple helix ${ }^{[50]}$.

A similar mechanism has been suggested to account for the induction of permeability changes in vesicular membranes of high surface charge density by electric impulses. Thus the displacement of the screening ionic atmosphere increases the mean distance between equally charged ionic groups on the membrane surface. The result is a permeability increase in the membrane. In this way we have interpreted the observation that electric impulses release biogenic amines from suspended chromaffin granules ${ }^{[51]}$.

The electric impulses applied to solutions of the $(\mathrm{A} \cdot 2 \mathrm{U})$ complex and to vesicle suspensions are comparable to the voltage pulses of the action potentials in nerve membranes. During excitation the electric field present across the nerve membrane changes in direction and intensity from about $-70 \mathrm{kV} / \mathrm{cm}$ to about $+50 \mathrm{kV} / \mathrm{cm}$. We may assume that large electric fields act not only across the membrane but also in close proximity to it and (transiently) across the synaptic junction. Hence, any polyvalently charged system exposed to these fieids could be affected in a similar way as the $(\mathrm{A} \cdot 2 \mathrm{U})$ complex or the chromaffin granules.
Electrically induced conformational changes in macromolecules and membranes have been discussed as a possible mechanism for electrically controlled regulatory processes in general and for a recording of electric signals in particular. With this in mind we may consider directed structural transitions induced by electric impulses in metastable polyelectrolyte systems as model reactions for the process of imprinting nerve impulses in the neuronal structures involved in a possible physical record of memory.

It is known from physiological and biochemical studies that nerve stimulation accelerates the metabolism of innervated cells; nerve activity, for instance, enhances RNA and protein synthesis. The mechanism of coupling between electrical activity and chemical reaction is still unknown. In view of the hysteresis capacity of rRNA which might be electrically affected in a similar way as in the A-U system, the question is raised whether ribosomal activity may be regulated via $\mathrm{rRNA}$ metastabilities by the electrical activity of the cell.

Other cellular processes, too, are influenced by nerve activity. Thus the secretion of hormones and neuroeffectors (transmitter substances) is appreciably increased during nerve stimulation. The depolarization voltage which leads to increased hormone secretion in the chromaffin cell is comparable to the initial intensity of the voltage pulses which in vitro cause outflow of neuramines from the suspended chromaffin granules. It is therefore suggestive to assume a mechanism according to which nerve activity controls directly the intensity and the extent of neurosecretion by permeability changes in the membranes of the storage vesicles transiently attached to the nerve membrane.

Furthermore, it is suggested that the elementary processes of synaptic facilitation in the functional development of the central nervous system involve permeability changes combined with neuroeffector release induced by nerve impulses in membranes.

It is worth mentioning that a threshold value of the impulse intensity of about $18 \mathrm{kV} / \mathrm{cm}$ was observed for the release of conformational transitions in the (A.2U) model system as well as for the induction of permeability changes in the vesicle membrane. The threshold value of the depolarization voltage necessary to release action potentials in nerve membranes is about $20 \mathrm{mV}$; for an average membrane thickness of $100 \AA, 20 \mathrm{mV}$ correspond to a field intensity of $20 \mathrm{kV} / \mathrm{cm}$. The similarity of these threshold voltages has revived the discussion on the chemical control of the ion flows underlying the action potentials. In line with this idea, conformational changes induced by impulses in membrane components are suggested as a mechanism for the activation of acetylcholine ${ }^{[53]}$. The relatively large amounts of heat accompanying the action potential imply the participation of chemical reactions in nerve activity. We note that chemical reactions and conformational changes which proceed irreversibly are accompanied by heat evolution due to dissipation of free energy. In this context it is of interest that during a depolarization-polarization cycle of the nerve membrane there is net heat production $^{[53]}$. It is a moot question whether this heat is caused by membrane components which change their conformation along a hysteresis loop (cf. the hysteresis observed by Tasaki in squid axon membranes ${ }^{[14]}$. 


\section{Hysteresis as a Basis of Chemical Oscillations}

The circular-vectorial property of hysteresis can be the basis for chemical oscillations. Microscopic chemical oscillations are of fundamental importance for a mechanistic interpretation of biological clocks ${ }^{[10.54]}$.

The principle of molecular hysteresis-based periodicity is the coupling of a chemical reaction flux with a diffusional process. The condition for the development of oscillation in this case is hysteresis, either in the reaction flux or in the diffusional flux. For instance, a periodic change in the concentration of a reaction product will be caused by a substance which participates both in a chemical reaction catalyzed by a "hysteretic" enzyme, and in a diffusion process (which may be controlled by a membrane). (Theoretically, coupling between auto-catalysis and enzyme reaction can also lead to oscillations; cf., e.g., ${ }^{[55]}$.)

Periodic changes based on chemico-diffusional flow coupling have been known for a long time in the heterocatalysis of gas reactions on metal surfaces (see, e.g., ${ }^{[56]}$ ). However, it has only recently been considered likely that such periodic processes may also occur on the level of single catalytically active molecules in homogeneous solution. In the halophilic malate-dehydrogenase we encounter an example of such a hysteretic catalyst ${ }^{[43]}$.

Periodic changes in diffusional flows can be caused by membranes which change their permeability along a hysteresis loop. The cell envelopes of Dead Sea halobacteria could change their permeability as a function of salt concentration in this way, if the structural change indicated in the proton binding hysteresis could cause permeability changes in the cell membrane. The experimental results of Ginzburg et al ${ }^{[57]}$ indicate that the Dead Sea halobacteria periodically change their salt content. Further studies may elucidate whether there is a correlation between membrane hysteresis and the hysteretic activity of the enzymes, causing the "salt clock" of these organisms.

The principle of hysteretic chemico-diffusional coupling may be (qualitatively) described with the aid of an enzyme which becomes active by substrate binding at a critical substrate concentration $S_{2}$. The substrate $S$ is assumed to flow at a constant rate in the reaction space; i.e., the diffusional flow, $J_{\mathrm{d}}$, of $\mathrm{S}$ is constant

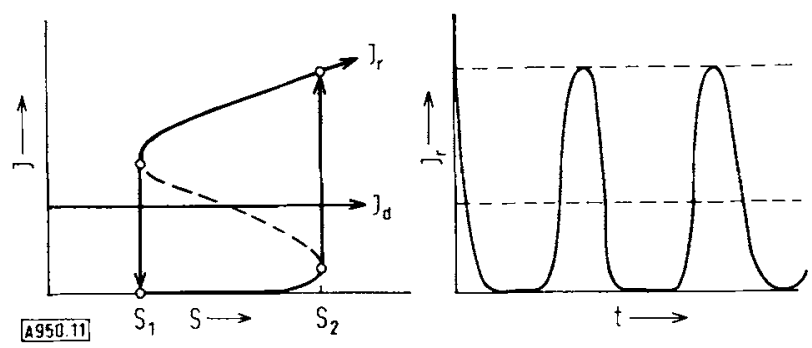

Fig. 11. Coupling scheme of transforming stationarity into periodicity by hysteretic chemico-diffusional coupling. $\mathrm{S}$, substrate concentration; $J_{\mathrm{d}}$, diffusional flux; $J_{r}$, reaction flux.

In the schematic representation of Fig. 11, it is seen that for $\mathrm{S}>\mathrm{S}_{2}$ the reaction flux $J_{\mathrm{r}}(\mathrm{S})$ is larger than $J_{\mathrm{d}}$. The breakdown of substrate is therefore faster than the influx of $S$. Thereby the substrate level in the reaction cell will sink until, at a critical concentration $S_{1}$, the enzyme becomes inactive. Now the reaction flux is smaller than the diffusional influx and substrate may accumulate until $\mathrm{S}>\mathrm{S}_{2}$. At $\mathrm{S}_{2}$ the cycle can start again. The result is a periodic change of the reaction flux and therefore an oscillation in the product concentration ${ }^{[10]}$.

In a similar way, a hysteretic permeability change in a membrane may transform a stationary flow into a periodic one. In this transducer function lies the fundamental significance of hysteretic macromolecules and membranes.

Whereas classical biology characterizes living organisms by stationary states (homeostasis), modern theories attempt to come closer to the actual dynamic behavior of biological organizations by a description which involves a quasi-stationary condition, with oscillations around a steady average $^{[58\}}$. Hysteresis represents a simple and energetically favorable mechanism for transducing stationarity into periodicity on a molecular level.

\section{Closing Remarks}

The discovery of conformational metastability in nucleic acids, proteins, and membranes has led to a new cybernetic approach to a dynamic interpretation of cellular behavior. It is worth noting that the dynamics of biological organization and function may be described by a general network theory recently developed by Oster, Perelson, and Katchalsky. In this approach, called network thermodynamics, "transducers" have been introduced as the coupling units between the network elements. It appears that macromolecules and membranes whose structure and function involve metastable states are particularly suited to act as elementary cybernetic units for "transducing" processes in general and for regulatory and memory reactions in particular. Metastable polyelectrolyte systems capable of storing "information" imprinted by ionic fluxes or electrical "activity" would be of significance for fast information processing with low energy expenditures.

In a dynamic picture of cellular organization and function there seems to be little room for perfect stability characterizing ideal equilibrium states. Even simple stationarity appears to be insufficient for a general description of central biological processes such as regulation and control of enzyme catalysis or membrane transport, information processing, or selforganization ${ }^{[60,61]}$. I believe that nonequilibrium approaches involving metastable states and molecular hysteresis may contribute to a better understanding of the complex dynamics of fundamental phenomena of life.

I would like to thank Arnold Revzin for critical comments regarding the manuscript and the Stiftung Volkswagenwerk for a research grant at the Weizmann Institute of Science in Israel.

Received: March 5, 1973 [A 950 IE]

German version: Angew. Chem. 85, 430 (1973)

[1] A. Katchalsky, Endeavour 12 (4), 90 (1953)

[2] A. Katchalsky, Biophys. J, 4, 9 (1964) 
[3] J. Stauff: Kolloidchemie. Springer-Verlag, Berlin 1960.

[4] D. H. Everett in E. A. Flood: The Solid Gas Interface, Vol. 2. Marcel Dekker, New York 1967, p. 1055

[5] A. Ewing, Proc. Roy. Soc. London 33, 21 (1881); Phil. Trans. Roy. Soc. London 523 (1885).

[6] R. A. Cox, A.S. Jones, G. E. Marsh, and A. R. Peacocke, Biochim. Biophys. Acta 21, 567 (1956)

[7] A. Revzin, E. Neumann, and A. Katchalsky, J. Mol. Biol., in press. [8] E. Neumann and A. Katchalsky, Ber. Bunsenges. Phys. Chem. 74, $868(1970)$.

[9] A. Katchalsky in S. Devons: Biology and the Physical Sciences. Columbia University Press, New York 1969, p. 267.

[10] A. Katchalsky and R. Spangler, Quart. Rev. Biophys. 1, 127 (1968).

[11] A. Katchalsky and E. Neumann, Int. J. Neurosci. 3, 175 (1972).

[12] A. Katchalsky and $\boldsymbol{G}$. Oster in D. C. Testeson: The Molecular Basis of Membrane Function. Prentice-Hall, Englewood Cliffs, N. J. 1969, p. 1.

[13] A. Sollberger: Biological Rhythm Research. Elsevier, Amsterdam 1965.

[14] I. Tasaki, W. Barry, and L. Carnay in F. Snell et al.: Physical Principles of Biological Membranes. Gordon-Breach, New York 1970 , p. 17

[15] H. R. Clark and A. Strickholm, Nature 234, 470 (1971).

[16] G. Adam in F. Snell et al.: Physical Principles of Biological Membranes. Gordon-Breach. New York 1970, p. 35.

[17] Cf., e.g.: S. Lifson and B. H. Zimm, Biopolymers $l, 15$ (1963).

[18] E. Neumann and T. Ackermann, J. Phys. Chem. 73, 2170 (1969).

[19] F. M. Pohl, Angew. Chem. 84, 931 (1972); Angew. Chem. internat. Edit. 11,894 (1972)

[20] H. Träuble, Naturwissenschaften 58, 277 (1971).

[21] T. L. Hill, J. Chem. Phys. 17, 520 (1949).

[22] G. Borelius, Ann. Phys. (Leipzig) 20, 57 (1934); Proc. Phys. Soc. London 49, 77 (1937).

[23] A. Katchalsky and P. F. Curran: Nonequilibrium Thermodynamics in Biophysics. Harvard University Press, Cambridge, Mass. 1965.

[24] I. Prigogine: Étude Thermodynamique des Processus Irreversibles. Desour, Liège 1947

[25] A. Katchalsky, A. Oplatka, and A. Litan in T. Hayashi and A. G. Szent-Györgyi: Molecular Architecture in Cell Physiology. PrenticeHall, Englewood Cliffs, N. J. 1966, p. 3; A. Katchalsky and A. Oplatka, Neurosci. Res. Symp. Sum. 1, 352 (1966).

[26] Z. Alexandrowices and A. Katchalsky, J. Polymer Sci. A1, 323 ! (1963).

[27] G. Felsenfeld and A. Rich, Biochim. Biophys. Acta 26, 457 (1957).

[28] R. C. Warner and E. Breslow, Proc. 4th Int. Congr. Biochem. Vienna $9,157(1958)$.

[29] $R$. F. Steiner and $R$. F. Beers, Jr., Biochim. Biophys. Acta 33, 470 (1959).

[30] R. A. Cox, Biochim. Biophys. Acta 68, 401 (1963).

[31] V. Vetterl and W. Guschlbauer, Arch. Biochem. Biophys. 148, 130 (1972).
[32] H.J.Hinz, O.J. Schmitz, and T. Ackermann, Biopolymers 7, 611 (1969).

[33] M. Spodheim and E. Neumann, Progr. Rep. Weizmann Inst. Israel (1972).

[34] W. Guschlbauer, D. Thiele, M.-T. Sarocchi, and C. Marck, Symp. Orléans 1972.

[35] D. Thiele and W. Guschlbauer, Biopolymers 8, 361 (1969).

[36] D. Barszcz and D. Shugar, Eur. J. Biochem. 5, 91 (1968).

[37] E. M. Gottschalk, E. Kopp, and A. G. Lezius, Eur. J. Biochem. 24,168 (1971)

[38] J. Pitha, P. M. Pitha, and P. O. P. Ts'o, Biochim. Biophys. Acta $204,39(1970)$

[39] Cf, e.g.: F. Travers, A. M. Michilson, and P. Douzou, Biochim. Biophys. Acta 199, 29 (1970)

[40] R. A. Cox u. A. Katchalsky, Biochem. J. 126, 1039 (1972),

[41] T. M. Schuster, B. R. Jennings, and G. Spach in: Nobel Symp. 11. Almqvist \& Wiksell, Stockholm 1969

[42] Cf, e.g.: S. N. Timasheff in A. Veis: Biological Polyelectrolytes. Marcel Dekker, New York 1970.

[43] M. Metarech and E. Neumann, Progr. Rep. Weizmann Inst. Israel (1972).

[44] E. Neumann, to be published.

[45] G. Weisbuch and E. Neumann, Biopolymers, in press.

[46] D. H. Everett, Trans. Faraday Soc. 51, 1551 (1955).

[47] J. A. Enderby, Trans. Faraday Soc. 51, 835 (1955).

[48] Cf., e.g.: E. Kneller: Ferromagnetismus. Springer-Verlag, Berlin 1962

[49] L. Boltzmann, Poggendorfs Ann. Erg. 7, 624 (1876).

[50] E. Neumann and A. Katchalsky, Proc. Nat. Acad. Sci. U. S. 69 , 993 (1972).

[51] E. Neumann and K. Rosenheck, J. Membrane Biol. 10, 279 (1972).

[52] E. Neumann, D. Nachmansohn, and A. Katchalsky, Proc. Nat. Acad. Sci. U. S. 70, 727 (1973)

[53] B. C. Abbott, A. V. Hill, and J.V. Howarth, Proc. Roy. Soc. London B 148, 149 (1958); J. V. Howarth, R. D. Keynes, and J. M. Ritchie, J. Physiol. (London) 194, 745 (1968).

[54] A. Katchalsky; Neurosci. Res. Program Bull. 9, 397 (1971).

[55] B. Edelstein, J. Theor. Biol. 29, 57 (1970).

[56] H. Beusch, P. Figuth, and E. Wicke, Chem.-Ing.-Tech. 44, 445 (1972) [57] M. Ginzburg, L. Sachs, and B. Z. Ginzburg, J. Gen. Physiol. 55, $187(1970)$.

[58] A. Katchalsky in G. C. Quarton, T. Melnechuk, and F. O. Schmitt: The Neurosciences, Vol. 1. The Rockefeller Univ. Press, New York 1967, p. 326.

[59] G. Oster, A. Perelson, and A. Katchalsk\}, Nature 234, 393 (1971); Quart. Rev. Biophys., in press.

[60] M. Eigen, Naturwissenschaften 58, 465 (1971).

[61] 1. Prigogine and G. Nicolis, Quart. Rev. Biophys. 4, 107 (1971) 